\title{
Kinematic Synthesis And Optimum Selection Of Planar Straight Line Mechanism
}

\author{
V. V. Pharate, S. M. Patil, O. M. Prajapati, S. P. Patil \\ (Department Of Mechanical Engineering, M. E. S. College Of Engineering, S. P. Pune University, India)
}

\begin{abstract}
This paper presents the study of mechanisms and methods to synthesize. In this article three planar straight line mechanisms are developed and designed using type, number and dimension synthesis. Calculations of their kinematics are done by vector loop equations and freudenstein's equations. Spread sheets are developed for the relation between input and output angles, and the travel distance for corresponding input. Selection of one mechanism which proves to be the best for given problem statement is done using relative point method. This method takes into consideration all the parameters necessary for the application.
\end{abstract}

Keywords : Freudenstein equation, Kinematic synthesis, Planar Mechanisms, Straight line mechanism.

\section{Introduction}

In a planar mechanism, all of the relative motions of the rigid bodies are in one plane or in parallel planes. Some of the highlighted advantages of planar mechanism over spatial mechanisms are that the working of planar mechanism is in a single plane, static and dynamic analysis is comparatively easy, any kind of error estimation [1] can be done efficiently. Such a planar straight line mechanism can be used for different industrial, commercial and construction purposes such as stacking or piling of finished products in industries and storage facilities, on construction sites for aligning of members etc. [2]. A set of such planar mechanisms are designed and developed using dimension, number and type synthesis for a given travel length in an approximate straight line motion. Type synthesis is the first step which deals with the types of elements used to transmit motion in the mechanisms. Number synthesis is the second step in the process of mechanism design which deals with determining the number of degree of freedom and the number of links and joints required. Third step is the Dimensional synthesis which deals with the determination of kinematic dimensions (link lengths, offsets, etc.) of the given mechanism to suffice the required characteristic motion. Graphical as well as analytical methods are available for dimensional synthesis. The choice of the method depends largely on the type of problem to be solved. The mechanisms are developed using this synthesis are Eight bar straight mechanism, Bell crank mechanism, Compound Scissor's mechanism for travel length ranging from $200 \mathrm{~mm}$ to $300 \mathrm{~mm}$ with linear and angular tolerance of $\pm 2.5 \mathrm{~mm}$ and $\pm 0.5^{\circ}$ respectively. The motion required is straight line for lowering a platform of length $250 \mathrm{~mm}$. The first two mechanisms are rather approximately straight lined but are well within the specified tolerance. Whereas the compound Scissor's mechanism is a perfectly straight line mechanism. These mechanisms are developed in CATIA V5 software to perform kinematics on the same. Each of the three mechanisms are provided with spread sheets including the link lengths, angular relations and the required travel lengths. From these three only one mechanism is selected which would satisfy the problem statement.

\subsection{Bell-Crank Mechanism}

\section{Mechanisms to be synthesized}

The mechanism shown in Fig. 1 is approximately straight line mechanism, consisting four bell-cranks, the angles of the bell-cranks are finalized using iteration method to give least possible linear deflection and the angles came out to be $123^{\circ}$ and $81^{\circ}$. The mechanism is so constraint to get one degree of freedom. The input output angle relationships are calculated using vector looping equation. From Fig.2 the vector loop equation can be written as $R_{1} \mathrm{e}^{\mathrm{j} \Theta 1}+\mathrm{R}_{2} \mathrm{e}^{\mathrm{j} \Theta 2}+\mathrm{R}_{3} \mathrm{e}^{\mathrm{j} \Theta 3}+\mathrm{R}_{4} \mathrm{e}^{\mathrm{j} \Theta 4}=0$, Where $\mathrm{R}_{1}, \mathrm{R}_{2}, \mathrm{R}_{3}, \mathrm{R}_{4}$ are lengths and $\Theta_{1}, \Theta_{2}, \Theta 3, \Theta 4$ are angles of link 1, 2, 3, 4 with respect to link 1. $\Theta_{2}$ is input angle and $\Theta_{4}, \Theta_{3}$ are the output angles. The governing equation of $\Theta_{2}$ and $\Theta_{4}$ is $2.67=10\left(\cos \Theta_{2}-\cos \Theta_{4}\right)+1.6 \cos \left(\Theta_{4}-\Theta_{2}\right)$. Using freudenstein's equations [3,4,5], $K_{1} \cos \Theta_{4^{-}} \mathrm{K}_{2} \cos$ $\Theta_{2}+K_{3}=\cos \Theta_{2} \cos \Theta_{4+} \sin \Theta_{2} \sin \Theta_{4}$. Then the relation between $\Theta_{2}$ and $\Theta_{4}$ is $\Theta_{4}=2 x \tan ^{-1} \frac{-B \pm \sqrt{B^{2}-4 A C}}{2 A}$. Similarly, the relationship between $\Theta_{2}$ and $\Theta_{3}$ can be derived. 


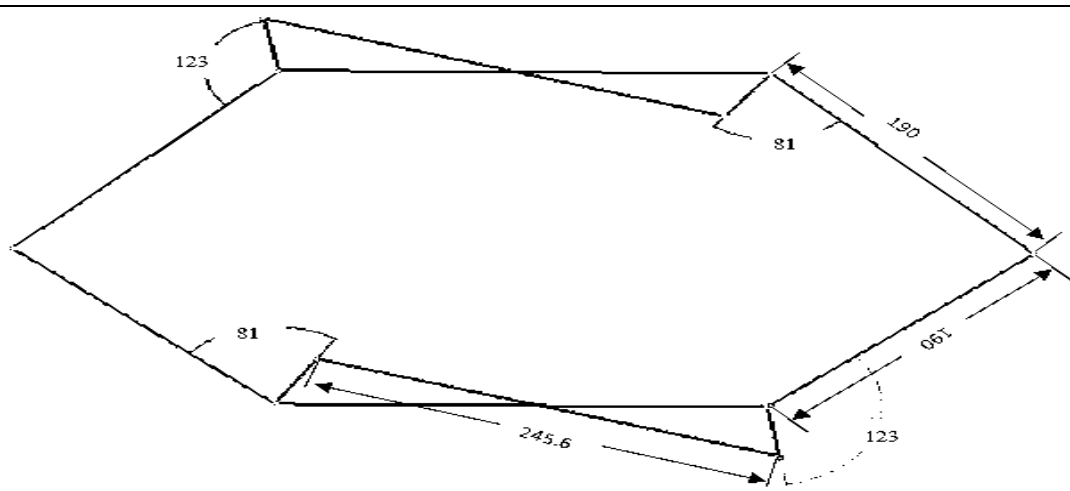

Fig. 1 Bell-crank mechanism line diagram

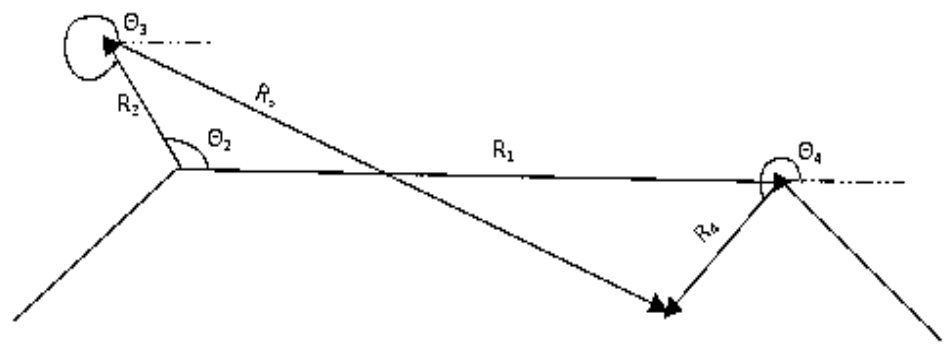

Fig. 2 Vector loop diagram for bell-crank

Travel length with respect to input angles is calculated using equation $\mathrm{L}=2 \times 190 \times \cos \Theta_{\mathrm{L}}$ where $\Theta_{\mathrm{L}}=$ $\Theta_{2}-90^{0}$, and summarized in Table 1 .

Table 1. Input \& output angular relationship with travel length of bell crank mechanism

\begin{tabular}{|c|c|c|}
\hline Input Angle $\Theta_{2}$ (Degrees) & Output Angle $\Theta_{4}$ (Degrees) & $\begin{array}{c}\text { Travel Length L } \\
(\mathrm{mm})\end{array}$ \\
\hline 80 & 255.3 & 156.2 \\
\hline 90 & 245.5 & 218.1 \\
\hline 100 & 236.1 & 272.9 \\
\hline 70 & 265.4 & 90.2 \\
\hline 65 & 270.6 & 55.9 \\
\hline 75 & 260.3 & 123.8 \\
\hline
\end{tabular}

\subsection{Eight Bar Straight Line Mechanism}

Eight bar straight line mechanism is obtained by combining tow five bar geared mechanisms with straight line motion as shown in Fig. 3. five bar geared mechanisms has five links and five joints giving it a resulting degree of freedom equals to 2 . Therefore 2 inputs will be required. This can be done by using a gear pair with a gear ratio of one so that both the inputs will be synchronized without any phase difference and resulting in desired straight line motion. The kinematics of the mechanism can be done on any one of the two five bar to find out the behavior of the whole eight bar mechanism to minimize the calculations. In addition to this the degree of freedom of the combined mechanism is equal to one. So a single input is more than enough for the desired output. The input output angle relationships are calculated using vector looping equation similar to the Bell-Crank mechanism, the only difference is there are 5 links which resulting three unknowns i.e. $\Theta_{3}, \Theta_{4}$, $\Theta_{5}[5,6]$. Since vector loop equation can be solved for only two unknowns. Combined mechanism needs another equation to solve the system [7,8]. As it's a geared five bar linkage, there exists a relationship between two geared links. Two factors determine response of link five with respect to link two, namely the gear ratio $\lambda$ and the phase angle $\Phi$. It can be obtained by the relation $\Theta_{5}=\lambda \Theta_{2+} \Phi$. For this mechanism gear ratio $\lambda=1$ and Phase angle $\Phi=-120^{\circ}$. Using Fig. 4, The vector loop equation $[1,3]$ can be written as $R_{1} \mathrm{e}^{\mathrm{j} \Theta 1}-\mathrm{R}_{2} \mathrm{e}^{\mathrm{j} \Theta 2}-\mathrm{R}_{3} \mathrm{e}^{\mathrm{j} \Theta 3}+\mathrm{R}_{4} \mathrm{e}^{\mathrm{j} \Theta 4}+\mathrm{R}_{5}$ $\mathrm{e}^{\mathrm{j}(\lambda \Theta 2+\Phi)}=0$. Solving the vector loop equation, relations between $\Theta_{2} \& \Theta_{3}$ or $\Theta_{2} \& \Theta_{4}$ are obtained. The relation between $\Theta_{2}$ and $\Theta_{4}$ is $\Theta_{4}=2 \times \tan ^{-1} \frac{-E \pm \sqrt{E^{2}-4 D F}}{2 D}$. Whereas the relation between $\Theta_{2}$ and $\Theta_{3}$ is $\Theta_{3}=2 \mathrm{x} \tan$ $\frac{1-M \pm \sqrt{M^{2}-4 L N}}{2 L}$ Output angle and travel length for corresponding input angle is calculated \& summarized in Table 2. 

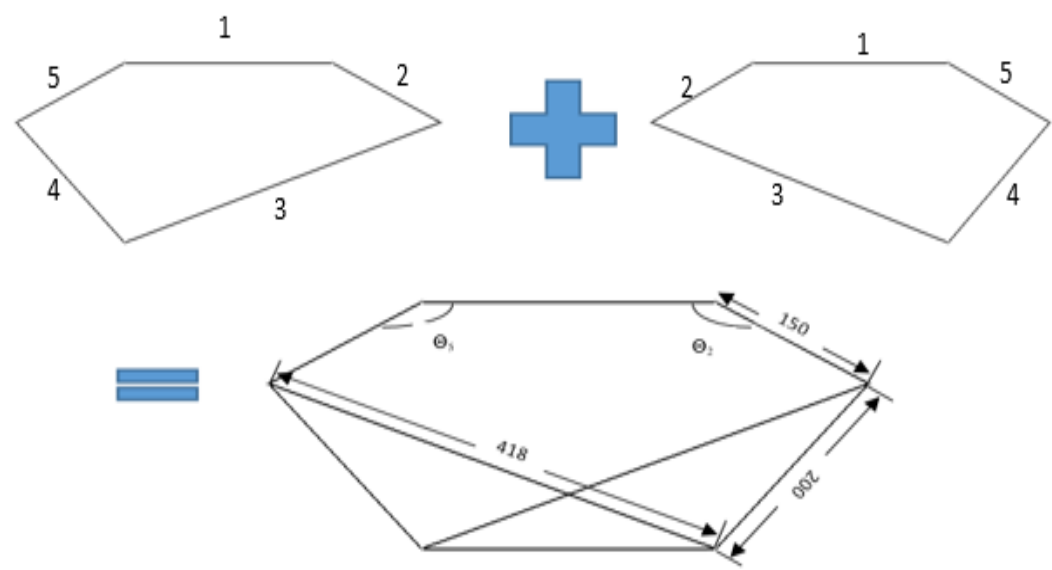

Fig. 3 Eight bar straight line mechanism

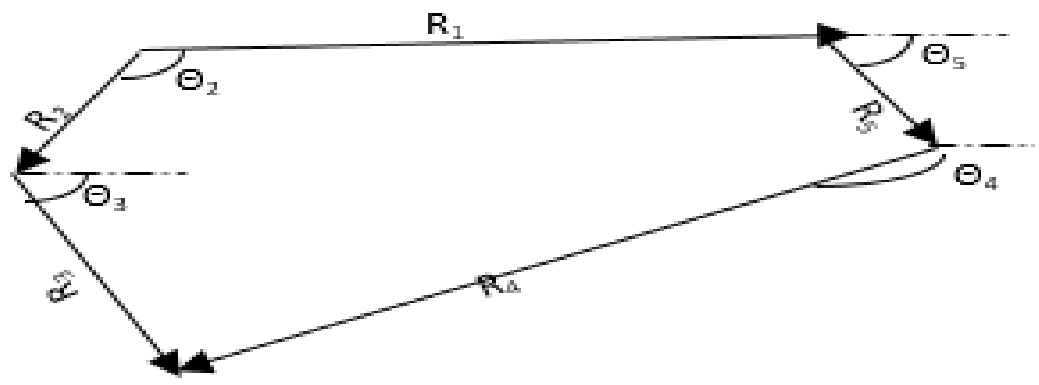

Fig.4 Vector loop diagram for eight bar mechanism

\begin{tabular}{|c|c|c|}
\hline \multicolumn{3}{|c|}{ Table 2. Input \& Output Angular Relationship with Travel Length for Eight Bar Mechanism } \\
\hline Input angle (Degree) & Output angle (Degree) & Travel length (mm) \\
\hline 140 & 146.8 & 261.5 \\
\hline 160 & 159.7 & 196.3 \\
\hline 130 & 141.1 & 291.2 \\
\hline 135 & 143.8 & 276.1 \\
\hline 145 & 245.3 & 149.9 \\
\hline
\end{tabular}

\subsection{Compound Scissor's Mechanism}

A Scissor's Mechanism is a perfectly straight line mechanism. mechanism is most suitable for the purpose of straight line travel [2]. The mechanism has a combination of ternary linkages and sliding part at both the input end and the output end. The main purpose of using Compound Scissor's Mechanism is travel and the platform length constrains. If a single mechanism is used for the travel of 200 to $300 \mathrm{~mm}$, the required link length will be more than $250 \mathrm{~mm}$ which is the platform length. In addition, when a single scissor's mechanism is used, the extended form of such arrangement is not capable of handling the load. This is because in extended state of mechanism, the slider is close to the fixed end leaving other end of platform unsupported. Now when a compound scissor's mechanism is used, the link lengths are within the platform length and both the platforms are equally supported. In Fig. 5, $\Theta_{2}$ be the input angle at the slider and $\Theta_{1}$ be the output angle. As the mechanism is symmetric, all the adjacent angles are equal.

$\Theta_{\mathrm{A}}+\Theta_{\mathrm{B}}+\Theta_{\mathrm{C}}+\Theta_{\mathrm{D}}=360^{\circ}$

Now, $\Theta_{\mathrm{A}}=\Theta_{\mathrm{C}} \& \Theta_{\mathrm{B}}=\Theta_{\mathrm{D}}$ as, $\mathrm{AB}=\mathrm{BC}=\mathrm{CD}=\mathrm{AD}$

$2 \Theta_{\mathrm{C}}+2 \Theta_{\mathrm{B}}=360$ or $\Theta_{\mathrm{B}}=180-\Theta_{\mathrm{C}}$

$\Theta_{\mathrm{B}}=2 \Theta_{2}$ and, $\Theta_{\mathrm{C}}=2 \Theta_{1}$ \& then $\Theta_{2}=90-\Theta_{1}$

The travel length of the mechanism is calculated by $\mathrm{Lm}=\mathrm{L} \sin \Theta_{2}$ where $\mathrm{Lm}=$ Travel of singular mechanism, $\mathrm{L}=$ Length of a linkage. Therefore, Total travel $=\mathrm{n} \mathrm{Lm}$. Where $\mathrm{n}=$ number of sets of singular mechanisms. Output angle and travel is calculated for corresponding input angle \& summarized in Table 3.

In Fig. 5, $\Theta_{2}$ be the input angle at the slider and $\Theta_{1}$ be the output angle. As the mechanism is symmetric, all the adjacent angles are equal.

$\Theta_{\mathrm{A}}+\Theta_{\mathrm{B}}+\Theta_{\mathrm{C}}+\Theta_{\mathrm{D}}=360^{\circ}$

Now, $\Theta_{\mathrm{A}}=\Theta_{\mathrm{C}} \& \Theta_{\mathrm{B}}=\Theta_{\mathrm{D}}$ as, $\mathrm{AB}=\mathrm{BC}=\mathrm{CD}=\mathrm{AD}$ 
$2 \Theta_{\mathrm{C}}+2 \Theta_{\mathrm{B}}=360$ or $\Theta_{\mathrm{B}}=180-\Theta_{\mathrm{C}}$

$\Theta_{\mathrm{B}}=2 \Theta_{2}$ and, $\Theta_{\mathrm{C}}=2 \Theta_{1} \&$ then $\Theta_{2}=90-\Theta_{1}$

The travel length of the mechanism is calculated by $\mathrm{Lm}=\mathrm{L} \sin \Theta_{2}$ where $\mathrm{Lm}=$ Travel of singular mechanism, $\mathrm{L}=$ Length of a linkage. Therefore, Total travel $=\mathrm{n} \mathrm{Lm}$. Where $\mathrm{n}=$ number of sets of singular mechanisms. Output angle and travel is calculated for corresponding input angle \& summarized in Table 3.

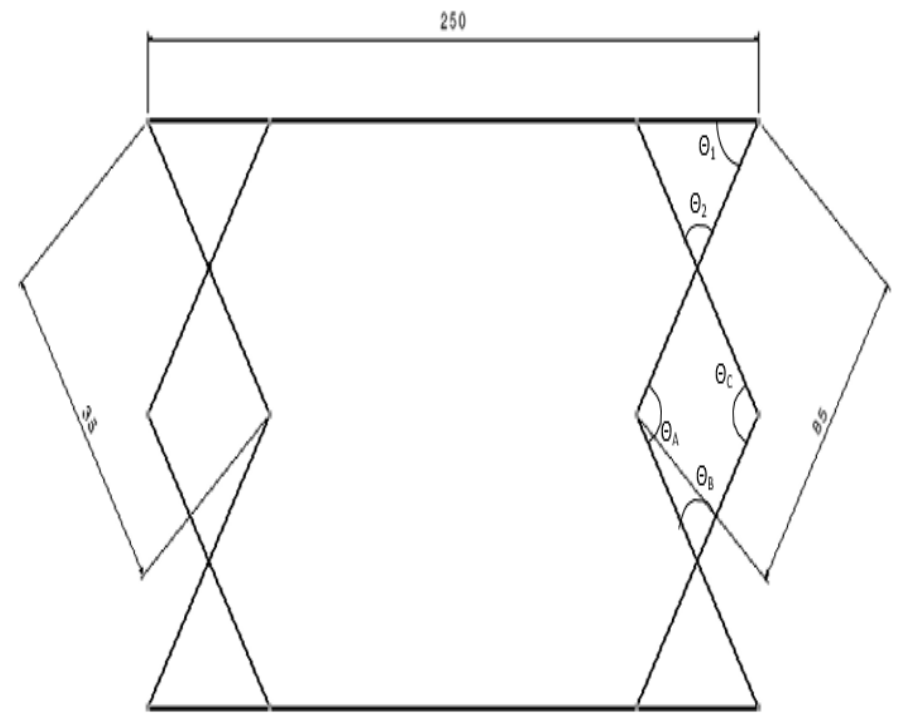

Fig.5 Compound scissor's mechanism

Table 3. Input output angular relationship with travel length for compound scissor's mechanism

\begin{tabular}{|c|c|c|}
\hline Input Angle (Degree) & Output Angle (Degree) & Total Travel of Mechanism (mm) \\
\hline 45 & 135 & 180.36 \\
\hline 55 & 125 & 208.93 \\
\hline 60 & 120 & 220.88 \\
\hline 75 & 105 & 246.34 \\
\hline 80 & 100 & 251.15 \\
\hline
\end{tabular}

\section{Relative point method for selection of optimum mechanism}

From the three mechanisms stated above only one mechanism is to be selected which is the optimum for application in current study. For this a selection technique must be adopted which will take into consideration of parameters like number of links, number of joints, type of joints etc. Considering this the method best suited for the selection optimum mechanism is Relative Point Method which has a governing formula

$$
\mathrm{P}=\frac{\mathrm{P} \max -\mathrm{Pa}}{\mathrm{P} \max -\mathrm{P}_{\min }} \mathrm{x} 10 \text { points }
$$

Where, $\mathrm{P}_{\max }=$ Maximum value of the parameter under consideration from all the mechanisms, $\mathrm{P}_{\min }=$ Minimum value of the parameter under consideration from all the mechanisms, $P_{a}=$ Actual Value of the considered mechanism. In this method the mechanism with maximum value of the given parameter is given zero points and the one with minimum value is given the highest points. The mechanism with intermediate value is given points according to the formula given above. Similarly, all the parametric points are calculated and sum of points for each mechanism is calculated. The mechanism with maximum points is chosen from the lot. Let us consider number of joints to be the parameter under consideration. $\mathrm{P}_{\max }=18 \& \mathrm{P}_{\min }=8$. Table 4 shows the sample point calculation for all mechanism based on number of joints.

Table 4. Number of joints for the three mechanisms \& points

\begin{tabular}{|l|c|c|}
\hline Mechanism & Number of joints & Points \\
\hline Bell Crank Mechanism & 10 & 8 \\
\hline Eight Bar Straight Line Mechanism & 8 & 10 \\
\hline Compound Scissor's Mechanism & 18 & 0 \\
\hline
\end{tabular}


Similar analysis can be done for all parameters like number of links, working envelope, angular tolerance, linear tolerance and number of revolute, sliding joint. Relative Point for all parameters are summarized in Table 5.

Table 5. Relative point summary for mechanism

\begin{tabular}{|l|c|c|c|}
\hline Mechanism & Bell Crank & Eight Bar & Compound Scissor's \\
\hline Number of Links & 10 & 7.5 & 0 \\
\hline $\begin{array}{l}\text { Number of revolute } \\
\text { joints }\end{array}$ & 8 & 10 & 0 \\
\hline $\begin{array}{l}\text { Number of Sliding } \\
\text { joints }\end{array}$ & 10 & 5 & 0.066 \\
\hline Working Envelope & 10 & 0 & 10 \\
\hline Linear tolerance & 2.86 & 0 & 18.066 \\
\hline Total & 40.26 & 22.5 & \\
\hline
\end{tabular}

From Table 5, Bell Crank Mechanism with maximum relative points is chosen, which is the most optimum choice for the application in current study.

\section{Conclusion}

Eight bar, Bell crank and Compound Scissor's Mechanisms are developed using type, number and dimensional synthesis. The Eight Bar Mechanism and Bell Crank Mechanisms are approximately straight line mechanisms but are well within the tolerance limits. Whereas Compound Scissors Mechanism is a perfectly straight line mechanism. All these mechanisms are designed using CATIA V5 and simulated to check straightness and tolerance constraints for the travel length $200 \mathrm{~mm}$ to $300 \mathrm{~mm}$. All the input \& output angle relations are calculated by vector loop equation and Freudenstein's equation. Relative point technique is used for selection of optimum straight line mechanism. This method highlights the main parameters of concern such as number of links, number and type of joints, linear tolerance and working envelope which gives bell crank mechanism as the optimum selection of straight line mechanism for given application in current study.

\section{References}

[1] A.Sedano, R.Sancibrian, Hybrid Optimization Approach for the Design of Mechanisms Using a New Error Estimator, Mathematical Problems in Engineering, 2012.

[2] F. Freudenstein, E R Maki, Creation of Mechanisms According to Kinematic Structure and Function, Environment \& planningB: urban analytics \& city science, 6(4), 1979, 375-391.

[3] F. Freudenstein, Approximate Synthesis of Four-Bar Linkages, ASME, 77, 1954, 853-861

[4] E.Soylemez, F. Freudenstein, Transmission optimization of spatial 4-link mechanisms, Mechanism and Machine Theory, 17(4), 1982, 263-283

[5] B. Roth, F. Freudenstein, Synthesis of path generating mechanism by numerical methods, Journal of Engineering for Industry, 85(3), 2011, 298-304.

[6] F. Roger, Gans, Analysis and Synthesis of planar mechanisms (Reed publishing (USA) Inc.: ButterworthHeinemann, 1991)

[7] Hongyu, Z. Ziyi, Design and Simulation Based on Pro/E for a Hydraulic Lift Platform in Scissors Type mechanism, Procardia Engineering, 16, 2011,772-781

[8] R. L Norton, Kinematics and Dynamics of machine elements (Boston, Mass.: McGraw-Hill, 2009) 